\title{
Characteristics of Intralimb Kinetic Coordination in the Lower Limbs During Gait in Patients with Hemiparesis Due to Stroke
}

\section{Yusuke Sekiguchi ( $\nabla$ yusuke.sekiguchi.b2@tohoku.ac.jp)}

Tohoku University https://orcid.org/0000-0002-9404-8659

\section{Dai Owaki}

Department of Robotics, Graduate School of Engineering, Tohoku University

\section{Keita Honda}

Department of Physical Medicine and Rehabilitation, Tohoku University Graduate School of Medicine Shin-ichi Izumi

Graduate School of Biomedical Engineering, Tohoku University

\section{Research}

Keywords: gait, ground reaction forces, hemiparesis, intralimb kinetic coordination, lower limbs

Posted Date: January 3rd, 2020

DOl: https://doi.org/10.21203/rs.2.19969/v1

License: (c) (i) This work is licensed under a Creative Commons Attribution 4.0 International License. Read Full License 
Characteristics of Intralimb Kinetic Coordination in the Lower Limbs During Gait in Patients with Hemiparesis Due to Stroke:

Yusuke Sekiguchi, ${ }^{1}$ Dai Owaki, ${ }^{2}$ Keita Honda, ${ }^{1}$ Shin-ichi Izumi ${ }^{1,3}$

${ }^{1}$ Department of Physical Medicine and Rehabilitation, Tohoku University Graduate School of Medicine, 2-1, Seiryo-machi, Sendai 980-8575, Japan

${ }^{2}$ Department of Robotics, Graduate School of Engineering, Tohoku University, 6-6-01Aoba, Aramaki, Aoba-ku, Sendai, 980-8579, Japan

${ }^{3}$ Graduate School of Biomedical Engineering, Tohoku University, 2-1, Seiryo-machi, Sendai 980-8575, Japan

\section{Correspondence Address:}

Yusuke Sekiguchi, RPT, PhD

Department of Physical Medicine and Rehabilitation,

Tohoku University Graduate School of Medicine

2-1, Seiryo-machi,

Sendai 980-8575,

Japan

Phone: 81-22-717-7338

Email: yusuke.sekiguchi.b2@tohoku.ac.jp 


\section{Abstract}

Background: The main objective of the present study is to investigate the relationship between the principal components (PCs) of the sagittal kinetic variables in the lower limb and the ground reaction forces (GRFs) during gait in patients with hemiparesis.

Methods: We recruited 21 patients with hemiparesis and 12 healthy controls. The 3dimensional (3-D) coordinates of 33 markers were measured with a 3-D motion analysis system operating at $120 \mathrm{~Hz}$ and force plates as the subjects walked along a 7-meter walkway. The correlation coefficients between the over-time series of PCs, which is calculated using principle component analysis (PCA), and GRFs were compared among the left side of the controls and the paretic side (PS) and non-PS of the patients by using analysis of variance (ANOVA).

Results: The correlation coefficient of the non-PS between the first PC and GRF in the anteroposterior-direction was significantly higher than that on the PS $(\mathrm{P}<0.05)$ and that of the non-PS in the vertical-direction was lower than the PS $(\mathrm{P}<0.05)$.

Conclusions: The results indicated that intralimb kinetic coordination on the PS plays an essential role in weight support in patients with hemiparesis, whereas the kinetic coordination on the non-PS plays a role in generation of propulsion. 
Keywords: gait, ground reaction forces, hemiparesis, intralimb kinetic coordination, lower limbs 


\section{Background}

Forward propulsion is a crucial biomechanical function for gait in patients with

hemiparesis. Specifically, forward propulsion on the paretic side during gait in patients with hemiparesis has been correlated with walking speed that strongly associated with community ambulation, quality of life, and mortality $[1,2,3]$. Moreover, it was also associated with the 6-Minute Walk Test distance, which can distinguish among home, limited community, and full community ambulators $[4,5]$. After a 12-week high-intensity locomotor training, changes in the forward propulsion on the paretic side during gait were correlated with that in the 6Minute Walk Test distance [4]. Therefore, forward propulsion is an essential gait parameter for walking ability and a primary target of post-stroke gait rehabilitation in patients with hemiparesis [6].

A previous study showed that several patients with hemiparesis increased the propulsion on the paretic side during gait without increasing the maximum ankle plantar flexor moment during speed modulation [7]. Hsio speculated that this strategy may be performed for fear of falling [7]. Allen demonstrated the difference in the contribution of hamstrings to propulsion on the paretic side during gait following rehabilitation between two patients with hemiparesis [8]. The findings demonstrated that increased joint moment only at a single joint does not neccesarily lead to increased forward propulsion during gait in patients with hemiparesis. 
A previous study defined intralimb kinetic coordination (in contrast to intralimb kinematic coordination) as an inter joint coordination based on kinetic variables [9]. Sadeghi et al. revealed that weight support and propulsion generation were important functions of intralimb kinetic coordination between the ankle and hip torques during gait in healthy controls, using principal component analysis (PCA) [10]. The principal components (PCs) indicate a simplification of the control of posture and locomotion due to a reduction in the available degrees of freedom by the central nervous system and functions of intralimb kinetic coordination $[10,33,34]$. However, there has been no study examining the functions, i.e. weight support and propulsion, of intralimb kinetic coordination in the lower limbs during gait in patients with hemiparesis. Propulsion generated by the paretic leg was also found to be sensitive to the hemiparetic severity level in patients with hemiparesis, identified using Brunnstrom motor recovery stages (a range of abilities to perform movements both inside and outside the extensor and flexor synergy patterns) $[11,12]$. Previous studies, which evaluated the muscle coordination during gait in patients with hemiparesis using electromyography (EMG), showed an impaired more complex motor modules [13, 14]. Moreover, the motor module related to propulsion in late stance during gait in patients with hemiparesis was combined with the other module related to body support during weight acceptance and deceleration of the leg at the end of swing and propulsion in early stance [13]. Therefore, intralimb kinetic coordination would suppress the propulsion generation due to the abnormal 
synergy pattern, which are combined with the several modules including the module related to propulsion.

Although the analysis of the muscle coordination using EMG of muscles in lower limb revealed the symptom of the abnormal synergy pattern in patients' gait in the previous study $[13,14]$, the kinetic mechanism of the abnormal synergy pattern remains unclear. The investigation into the kinetic mechanism of the abnormal synergy pattern using intralimb kinetic coordination could lead appropriate intervention for the abnormal synergy pattern by gait training, orthosis, and robots. In the clinical practice on stroke rehabilitation, evaluation of impaired intralimb kinetic coordination in these patients may be helpful in treating propulsion deficits and supporting weight during gait with an orthosis or a wearable robot worn at a single or several joints in the lower limb.

The objectives of the present study were as follows: 1) to investigate the difference in the intralimb kinetic coordination and loadings of each joint in the lower limbs on the paretic and nonparetic sides in patient with hemiparesis, and the left side (LS) of healthy controls and 2) to evaluate the relationship between the intralimb kinetic coordination in the lower limbs and weight support and propulsion in patients with hemiparesis. 


\section{Methods}

\section{Participants:}

21 patients with hemiparesis due to stroke (17 males and 4 females; age 30-74 years) and 12 healthy controls of comparable age (5 males and 7 females; age 31-78 years) were enrolled in the study. The demographic and clinical characteristics of the study subjects are shown in Table 1. The inclusion criteria for the subjects consisted of a first-time stroke due to either an ischemic or hemorrhagic supratentorial lesion and the ability to walk at least 7 meters without an assistive device. The exclusion criteria for the subjects with hemiparesis and controls consisted of the presence of brain stem or cerebellar lesions, higher brain dysfunction (which would skew the measurements), and orthopedic problems.

In patients with hemiparesis, the severity of the hemiparesis and the ability to perform movements outside the extensor and flexor synergy patterns were assessed using Brunnstrom's stages of recovery [11]. These tests were performed using standardized protocols by an experienced physical therapist (Y. S.). This study was conducted in accordance with the Helsinki Declaration. All participants provided written informed consent prior to data collection, and the Institutional Review Board approved the study.

\section{Gait analysis:}

The study subjects were asked to walk 7 meters for $2-10$ trials. The patients were instructed to walk at a self-selected comfortable pace without assistive devices, whereas the 
controls were instructed to walk at a slow pace. The results were the average of more than 5 strides in successful trials. In addition, 33 reflective markers were attached to 12 segments with adhesive tapes (Table 2). For all measurements, the coordinates of each reflective marker were measured using the MAC 3D System $(120 \mathrm{~Hz})$ (Motion Analysis Corporation, Santa Rosa, CA, USA). Ground reaction forces (GRF) data were acquired at a 1,200 Hz sampling rate using four $90 \mathrm{~cm} \times 60 \mathrm{~cm}$ force plates (Anima Corporation, Choufu, Tokyo, Japan). The 3-D coordinates and GRF data were smoothed using a bidirectional fourth-order Butterworth low-pass filter with a cut-off frequency of $20 \mathrm{~Hz}$ and $200 \mathrm{~Hz}$, respectively. A 12segment model based on anthropometric data, in accordance with Dumas [15], and comprised of feet, shanks, thighs, pelvis, thorax, upper arms, and forearms, was used. The kinematic data for each joint in the lower extremities were calculated using a joint coordinate system [16]. In addition, the lower extremity joint kinetics were estimated using the inverse dynamics [17]. All kinematic and kinetic data were time-normalized to $100 \%$ of 1 gait cycle. The representative parameters for gait in the patient group were extracted from the kinematic and kinetic data in accordance with the previous study by Kinsella [18]. The kinetic data were normalized by patient's body weight, and the parameters were calculated with customized software using Matlab (Mathworks Inc., Natick, MA). 


\section{Principle component analysis (PCA) using singular value decomposition:}

To investigate the differences in the intralimb kinetic coordination of the lower limbs and the loadings of each joint in the lower limbs of the paretic side (PS) and the non paretic side (non-PS) in the patient group, and the left side (LS) of the healthy controls, we conducted movement decomposition using PCA based on kinetic variables, i.e., joint moments [19, 20].

The kinetic variables can be expressed by writing the time-series data of the joint moments as a column in the matrix $R(\tau, t)$ as follows:

$$
R(\tau, t)=\left[\begin{array}{ccc}
\tilde{\tau}_{\text {hip }}\left(t_{1}\right) & \tilde{\tau}_{\text {knee }}\left(t_{1}\right) & \tilde{\tau}_{\text {ankie }}\left(t_{1}\right) \\
\tilde{\tau}_{h i p}\left(t_{2}\right) & \tilde{\tau}_{\text {knee }}\left(t_{2}\right) & \tilde{\tau}_{\text {ankie }}\left(t_{2}\right) \\
\vdots & \vdots & \vdots
\end{array}\right]
$$

where $\tilde{\tau}_{\text {hip }}\left(t_{j}\right), \tilde{\tau}_{\text {knee }}\left(t_{j}\right), \tilde{\tau}_{\text {ankie }}\left(t_{j}\right)$ denote the normalized moments at each $j^{\text {th }}$ step (\%) using the absolute maximum moments $\max \left\{\left|\tau_{i}\left(t_{j}\right)\right|\right\}$ during one period $[j: 0-100(\%)]$. PCA is conducted by finding the eigenvectors and eigenvalues of the covariance matrix of object data, e.g., $\operatorname{conv}(R(\tau, t))$. By using singular value decomposition (SVD), the eigenvectors $Z_{i}(\tau)$ of $\operatorname{conv}(R(\tau, t))$ can be easily calculated as follows:

$$
R(\tau, t)=\sum_{i} \lambda_{i} v_{i}(t) z_{i}(\tau)(2)
$$

where $\lambda_{i} v_{i}(t)$ represents time-series values with the corresponding eigenvectors $z_{i}(\tau)$.

Thus, the time-series data $R(\tau, t)$ of the joint moments can be decomposed into timeinvariant spatial patterns $z_{i}(\tau)$ and temporal characteristic patterns $\lambda_{i} v_{i}(t)$ of the corresponding spatial patterns $z_{i}(\tau)$. In addition, the percentage of variance (\%) for the $i^{\text {th }}$ 
PC was calculated using $\sum_{i}\left(\lambda_{i}\right)^{2} / \sum_{3}\left(\lambda_{i}\right)^{2}$. The reason for employing SVD was that SVD analysis can divide the time-series data of joint moments into spatial and temporal patterns of PCs (e.g., using Matlab functions), and therefore allows for the visualization of the contribution ratio of these spatial and temporal patterns for the corresponding gait at any given time.

\section{$\underline{\text { Statistical analysis: }}$}

The gait speed, stride time, and stride length between the patient group and the healthy controls were compared using unpaired $t$-tests. Differences in the spatiotemporal, representative kinetic, and PCA-related parameters along the LS of healthy controls and the PS and non-PS in the patient group were examined using a 1-way analysis of variance (ANOVA) followed by a Tukey's test. The correlation coefficients between the time series of the PCs and GRFs were compared among the LS of healthy controls, the PS, and the non-PS of the patient group using an ANOVA followed by a Tukey's test. The relationship between Brunnstrom's stages of recovery and scores of sensory function and PCA-related parameters were examined using the Spearman's rank-correlation coefficient. The significance level for all tests was set at $P<0.05$. All statistical tests were performed using the Statistical Package for the Social Sciences (SPSS), version 22.0 (SPSS Inc., Chicago, IL, USA). 


\section{Results}

All parameters during gait in the patient group and the healthy controls are shown in

Figure 1 and in Tables 3 and 4. There were no significant between-group differences in age, weight, or height.

\section{Gait speed and spatiotemporal parameters:}

There were no significant differences between the gait speeds of the 2 groups. Significant differences in the stance time $(P<0.05)$, swing time $(P<0.01)$, and single-leg stance time $(P$ $<0.01$ ) among the 3 groups were observed. Stance time on the PS of the patients was significantly shorter than that along the LS of healthy controls $(P<0.05)$. Swing time on the non-PS $\quad(P<0.01)$ and single support time on the PS were the shortest of the 3 groups $(P<$ $0.01)$.

\section{Kinetic parameters:}

There were significant differences in the maximum hip extension moment in the early stance $(P<0.01)$ and the maximum ankle plantar flexion moment $(P<0.01)$ in the stance phase among the 3 groups. There tended to be a difference in the maximum hip flexion moment in the stance phase, although it was not significant $(P=0.06)$. The maximum hip extension moment in the early stance for the nonparetic side in the patient group was the 
highest of the 3 groups $(P<0.05$ for both). For the paretic side, the maximum ankle plantar flexion moment in the stance phase was the lowest of the 3 groups $(P<0.05$ for both).

\section{PCA-related parameters:}

The PCA of the joint moments demonstrated that the first and second PCs accounted for the total variances of $82 \%$ and $17 \%$, respectively, for the LS of the healthy controls, $76 \%$ and $19 \%$, respectively, for the non-PS, and $81 \%$ and $17 \%$, respectively, for the PS. The first 2 PCs on the non-PS accounted for $95 \%$, which is lower than the percentage of variance of the first 2 PCs on the PS $(P<0.01)$ and the LS of the healthy controls $(P<0.05)$.

Significant differences in the loadings of the knee joint moment for the first and second PCs were observed $(P<0.05$ for both). The loadings of the knee joint moment for the first PC on the PS were lower than those on the non-PS $(P<0.05)$. Additionally, compared with the loadings of the knee joint on the LS of the healthy controls, those of the non-PS of the patients were significantly lower $(P<0.05)$.

The correlation coefficient of the non-PS between the time series of the first PC and GRF in the anteroposterior direction during gait was higher than that of the PS in the patient group $(P<0.05)$. The correlation coefficient for the PS $(P<0.05)$ and the LS of the healthy controls $(P<0.05)$ between the time series of the first PC and GRF in the vertical direction was higher than that for the non-PS. 


\section{Correlation between PCA-related parameters, Brunnstrom's stages of recovery:}

There were no significant correlations between the percentage of variance of the first PC $(\mathrm{r}=-0.14)$, second PC $(\mathrm{r}=0.15)$, and the first $2 \mathrm{PCs}(\mathrm{r}=0.15)$ on the PS and Brunnstrom's stages of recovery.

\section{Discussion}

This study demonstrates that the percentage of variance described by the first 2 components of kinetic variables in the lower limbs exceeded $95 \%$ of the total variance. These results show that the time series of kinetic variables during gait in healthy controls and patients with hemiparesis might follow a planar law in a 3-D space, similar to that of kinematic variables, as demonstrated in previous studies $[21,22,32]$. The planar law could reflect a centrally controlled behavior, above biomechanical constraints [21, 22]. Generally, the time series of PCs analyzed using PCA was characterized based on the description of each representative curve [23]. This study has identified the correlation coefficient between PCs composed of kinetic variables in the lower limbs and the GRF in healthy controls, as well as patients with hemiparesis, to elucidate the meaning of time series for each PC. A previous study reported that the first PC in healthy controls revealed the primary function of the hip and ankle joint moments in the lower limbs in order to prevent the body from collapsing [10]. Correlation coefficients between the first PC and GRF in a vertical direction for each of the 3 
groups were high in this study $(\mathrm{r}=0.62-0.76)$, which is in line with the interpretation of the previous study [10]. Additionally, the correlation coefficient between the first PC and GRF in the anteroposterior direction was also high, excluding that of the PS. To the best of our knowledge, this is the first study to clarify the function of intralimb kinetic coordination in the lower limbs during gait in patients with hemiparesis.

The findings suggest that the correlation coefficient between the first PC and GRF in the forward direction for the PS is low, whereas the the correlation coefficient in the vertical direction is high. A previous study also showed that the PS of patients with hemiplegic cerebral palsy exhibits greater leg stiffness, smaller landing angle, which formed between the vector connecting the body's center of mass to the ankle joint and another vector representing the vertical direction, and a higher ratio of forward kinetic energy for potential energy [24]. Similary, our previous study demonstrated that ankle joint stiffness in early stance that steeply increases the GRF in the vertical direction on the PS increased in patients with hemiparesis [25]. Therefore, these findings have supported that the impaired kinetic control through abnormal intralimb kinetic coordination would incline toward the vertical direction of the lower leg on the PS during gait. Moreover, several studies have showed that the wholebody angular momentum (WBAM) in the sagittal plane in the second half phase of gait cycle and that in the frontal plane in the single stance phase on the PS were higher than that in healthy controls $[26,27]$. WBAM in both sagittal and frontal planes is affected by the GRF in 
the vertical direction, whereas WBAM in only sagittal plane is affected by the GRF in the forward direction [35]. The facts suggests that GRF control through intralimb kinetic coordination in lower limb on the PS would plays essential role on the vertical direction during gait in patients with hemiparesis due to necessity of the strict control for the high WBAM in the sagittal and frontal planes.

For the non-PS, The correlation coefficient between the first PC and GRF in the anteroposterior and vertical directions were opposite to that of the PS. These results demonstrated that the kinetic coordination on the non-PS compensated for the decreased forward propulsion on the PS. In a study of the GRF in patients with hemiparesis, Bowden et al. found that the percentage of propulsion generated by the non-PS leg was higher than that by the PS leg [12]. Moreover, in a study involving EMG during gait in patients with hemiparesis, the plantar flexor activity during the push-off and early stance, as well as hamstring activity during the early stance on the non-PS, increased [28]. Therefore, results of these previous studies are similar to those of this study with regard to the non-PS. In addition, the percentage of variance in the first 2 PCs on the non-PS was lower than that on the PS and in healthy controls. Therefore, the kinetic control speculated to be exhibited by intralimb kinetic coordination in the lower limbs on the non-PS would require more motor modules because groups of muscles are activated together to compensate for propulsion occurring on the PS only and maintaining balance due to the instability of the PS leg in patients. 
This study also found that abnormal synergy patterns, evaluated using Brunnstrom's recovery stages, weakly correlated with the variance percentage of the first PC, second PC, and the first 2 PCs on the PS. Using Brunnstrom's stage for detection of the complex motor module during gait would be difficult due to synergy pattern abnormality (evaluated using Brunnstrom's stage), which does not represent an abnormal muscle activity during gait, but rather while sitting or standing.

This study has several limitations. First, there were no inclusion criteria related to the time since the neurologic event. A previous study found significant differences in the intralimb kinematic joint during gait between acute and chronic stages [29]. Therefore, the relationship between the time since the neurologic event and intralimb kinetic coordination should be investigated in future studies. Second, healthy controls in this study were not sexmatched to that in the patient group. Chow and Stokic found no differences in the intralimb kinematic coordination of the lower limbs during gait between healthy men and women in their study [30]. As the kinematic variable represents the movement characteristics resulting from force due to muscle activation, the intralimb kinetic coordination of the lower limbs during gait in men may also be similar to that in women. Therefore, sex differences appear not to affect the results of this study. Third, differences in kinetic data may be observed between a measured and more realistic walk in healthy controls, because they were asked to walk at a slow speed. However, walking speed influences the kinetic data in the lower limbs 
[31]. Therefore, an accurate comparison with the kinetic data in patients with hemiparesis should be made with those measured at a slow walking speed in healthy controls. Fourth, as the standard deviation for loadings of the knee moment on the first PC was high, the effect of knee moment on the first PC would not correspond to that of all patients with hemiparesis.

The types of knee moment patterns on the PS varied in the patient group. Therefore, the effect of knee moment pattern difference on the first PC should be investigated in future studies.

\section{Conclusions}

Our findings suggest the main task of intralimb kinetic coordination during gait in patients with hemiparesis by using PCA. The present investigation found that the correlation coefficient on the paretic side between the time series of the first PC and GRF in the anteroposterior direction during gait was lower than that of the nonparetic side for patients with hemiparesis. However, the correlation coefficient between the time series of the first PC and GRF in the vertical direction for the paretic side was higher than that of the nonparetic side. These findings indicate that the intralimb kinetic coordination of the lower limbs for the paretic side during gait in patients with hemiparesis would play an essential role in the support of weight but not for the generation of propulsion. 


\section{Acknowledgments}

We would like to thank the staff at the Department of Physical Medicine and

Rehabilitation at Tohoku University for their advice and assistance. This work was supported by JSPS KAKENHI, Grant-in-Aid for Early-Career Scientists B (18K17665), Scientific Research on Innovative Areas, "Understanding brain plasticity on body representations to promote their adaptive functions" $(26120007,26120008)$, and the NEC corporation.

\section{References}

1. Perry J, Garrett M, Gronley J, Mulroy S. Classification of walking handicap in the stroke population. Stroke. 1995;26:982-9.

2. Middleton A, Fritz SL, Lusardi M. Walking speed: the functional vital sign. J Aging Phys Act. 2015;23:314-22.

3. Studenski S, Perera S, Patel K, et al. Gait speed and survival in older adults. JAMA. 2011;305:50-8.

4. Awad LN, Binder-Macleod SA, Pohlig RT, Reisman DS. Paretic propulsion and trailing limb angle are key determinants of long-distance walking function after stroke. Neurorehabil Neural Repair. 2015;29:499-508.

5. Fulk GD, He Y, Boyne P, Dunning K. Predicting home and community walking activity poststroke. Stroke. 2017;48:406-11.

6. Roelker SA, Bowden MG, Kautz SA, Neptune RR. Paretic propulsion as a measure of walking performance and functional motor recovery post-stroke: A review. Gait Posture. 2019;68:6-14.

7. Hsiao H, Knarr BA, Higginson JS, Binder-Macleod SA. Mechanisms to increase propulsive force for individuals poststroke. J Neuroeng Rehabil. 2015;12:40.

8. Allen JL, Kautz SA, Neptune RR. Forward propulsion asymmetry is indicative of changes in plantarflexor coordination during walking in individuals with post-stroke hemiparesis. Clin Biomech (Bristol, Avon). 2014;29:780-6. 
9. Longworth JA, Chlosta S, Foucher KC. Inter-joint coordination of kinematics and kinetics before and after total hip arthroplasty compared to asymptomatic subjects. $\mathrm{J}$ Biomech. 2018;72:180-6.

10. Sadeghi H, Sadeghi S, Prince F, Allard P, Labelle H, Vaughan CL. Functional roles of ankle and hip sagittal muscle moments in able-bodied gait. Clin Biomech (Bristol, Avon). 2001;16:688-95.

11. Brunnstrom S. Recovery stages and evaluation procedures. In:Brunnstrom S, editor. Movement therapy in hemiplegia: a neurophysiological approach. New York: NY Harper \& Row; 1970. p. 34-55.

12. Bowden MG, Balasubramanian CK, Neptune RR, Kautz SA. Anterior-posterior ground reaction forces as a measure of paretic leg contribution in hemiparetic walking. Stroke. 2006;37:872-6.

13. Clark DJ, Ting LH, Zajac FE, Neptune RR, Kautz SA. Merging of healthy motor modules predicts reduced locomotor performance and muscle coordination complexity poststroke. J Neurophysiol. 2010;103:844-57.

14. Brough LG, Kautz SA, Bowden MG, Gregory CM, Neptune RR. Merged plantarflexor muscle activity is predictive of poor walking performance in post-stroke hemiparetic subjects. J Biomech. 2018;82:361-367.

15. Dumas R, Cheze L, Verriest JP. Adjustments to McConville et al. and Young et al. body segment inertial parameters. J Biomech. 2007;40:543-53.

16. Winter DA. Three-dimensional kinematics and kinetics. In: Winter DA, editor. Biomechanics and motor control of human movement 4th ed. Hoboken, NJ: John Wiley \& Sons Inc; 2009. p. 176-99.

17. Hammill J, Selbie WS, Kepple TM. Three-Dimensional Kinematics. In: Robertson DGE, Caldwell GE, Hamill J, Leeds KG, editors. Research Methods in Biomechanics. 2nd ed. United Kingdom: Human Kinetics; 2014. p. 35-60.

18. Kinsella S, Moran K. Gait pattern categorization of stroke participants with equinus deformity of the foot. Gait Posture. 2008;27:144-51.

19. Funato T, Aoi S, Oshima H, Tsuchiya K. Variant and invariant patterns embedded in human locomotion through whole body kinematic coordination. Exp Brain Res. 2010;205:497-511.

20. Troje NF. Decomposing biological motion: a framework for analysis and synthesis of human gait patterns. Journal of vision. 2002;2:371-87.

21. Cheron G, Bouillot E, Dan B, Bengoetxea A, Draye JP, Lacquaniti F. Development of a kinematic coordination pattern in toddler locomotion: planar covariation. Exp Brain Res. 2001;137:455-66. 
22. Ivanenko YP, d'Avella A, Poppele RE, Lacquaniti F. On the origin of planar covariation of elevation angles during human locomotion. J Neurophysiol. 2008;99:1890-8.

23. Sadeghi H, Prince F, Sadeghi S, Labelle H. Principal component analysis of the power developed in the flexion/extension muscles of the hip in able-bodied gait. Med Eng Phys. 2000;22:703-10.

24. Fonseca S, Holt K, Fetters L, Saltzman E. Dynamic resources used in ambulation by children with spastic hemiplegic cerebral palsy: relationship to kinematics, energetics, and asymmetries. Phys Ther. 2004;84:344-54; discussion 55-8.

25. Sekiguchi Y, Muraki T, Tanaka N, Izumi S. Relationship between activation of ankle muscles and quasi-joint stiffness in early and middle stances during gait in patients with hemiparesis. Gait Posture. 2015;42:348-53.

26. Honda K, Sekiguchi Y, Muraki T, Izumi SI. The differences in sagittal plane wholebody angular momentum during gait between patients with hemiparesis and healthy people. $\mathrm{J}$ Biomech. 2019;86:204-9.

27. Vistamehr A, Balasubramanian CK, Clark DJ, Neptune RR, Fox EJ. Dynamic balance during walking adaptability tasks in individuals post-stroke. J Biomech. 2018;74:106-15.

28. Raja B, Neptune RR, Kautz SA. Coordination of the non-paretic leg during hemiparetic gait: expected and novel compensatory patterns. Clin Biomech (Bristol, Avon). 2012;27:1023-30.

29. Milovanovic I, Popovic DB. Principal component analysis of gait kinematics data in acute and chronic stroke patients. Comput Math Methods Med. 2012;2012:649743.

30. Chow JW, Stokic DS. Intersegmental coordination scales with gait speed similarly in men and women. Exp Brain Res. 2015;233:3175-85.

31. Goldberg SR, Stanhope SJ. Sensitivity of joint moments to changes in walking speed and body-weight-support are interdependent and vary across joints. J Biomech.

2013;46:1176-83.

32. Chow JW, Stokic DS. Intersegmental coordination of gait after hemorrhagic stroke. Exp Brain Res. 2015;233:125-135.

33. Lacquaniti F, Grasso R, Zago M. Motor patterns in walking. News Physiol Sci. 1999;14:168-74.

34. Borghese NA, Bianchi L, Lacquaniti F. Kinematic determinants of human locomotion. J Physiol. 1996;494:863-79.

35. Silverman AK, Neptune RR. Differences in whole-body angular momentum between below-knee amputees and non-amputees across walking speeds. J Biomech. 2011;44:379-85. 


\section{Declarations}

\section{Ethics approval and consent to participate}

The present study was approved by the Ethics Committee of Tohoku University School of Medicine (2016-1-354). All participants provided written informed consent in accordance with ethical guidelines.

\section{Consent for publication}

All authors have approved the manuscript for publication.

\section{$\underline{\text { Availability of data and materials }}$}

The datasets analysed in the present study are not publicly available because of clinical policy but are available from the corresponding author on reasonable request.

\section{Competing interests}

The authors declare that they have no competing interests.

\section{Funding}

This work was supported by JSPS KAKENHI, Grant-in-Aid for Early-Career Scientists B (18K17665) and Scientific Research on Innovative Areas, "Understanding brain plasticity on body representations to promote their adaptive functions" (26120007, 26120008). $\underline{\text { Authors' contributions }}$ 
Yusuke Sekiguchi designed the study, collected data, analyzed data, and wrote the initial draft of the manuscript. Dai Owaki contributed to analysis and interpretation of data and assisted in the preparation of the manuscript. Kita Honda contributed to data collection, analysis of data, and interpretation of data. Shin-Ichi Izumi have contributed to interpretation of data and critically reviewed the manuscript.

\section{$\underline{\text { Acknowledgements }}$}

We would like to thank the staff at the Department of Physical Medicine and Rehabilitation at Tohoku University for their advice and assistance.

\section{Figure Captions}

Figure 1 - A typical first PC1 and GRFs in the vertical and anteroposterior directions during gait. The left, middle, and right figures indicate the PC1, the GRF in the anteroposterior direction, and the GRF in the vertical direction.

Figure 2 - A typical ankle, knee, and hip moment during 1 gait cycle. The left, middle, and right figures indicate the ankle, knee, and hip moments during 1 gait cycle. 
Figures

Fig.1

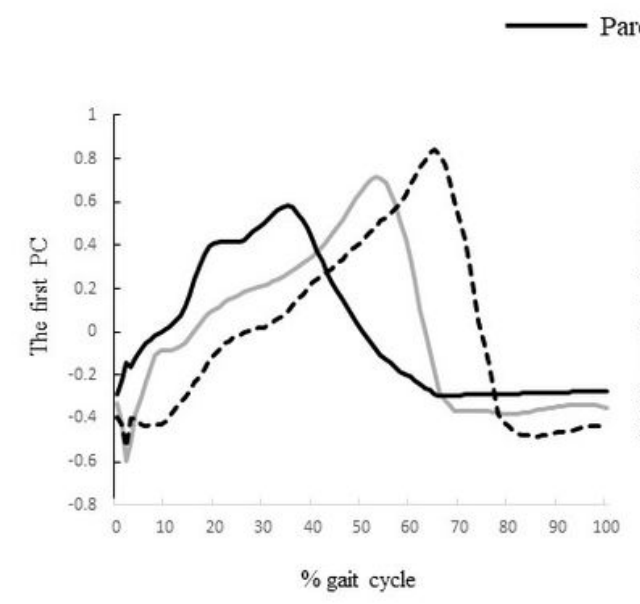

Paretic side $\quad$ - - - Non-Paretic side

Left side in Control
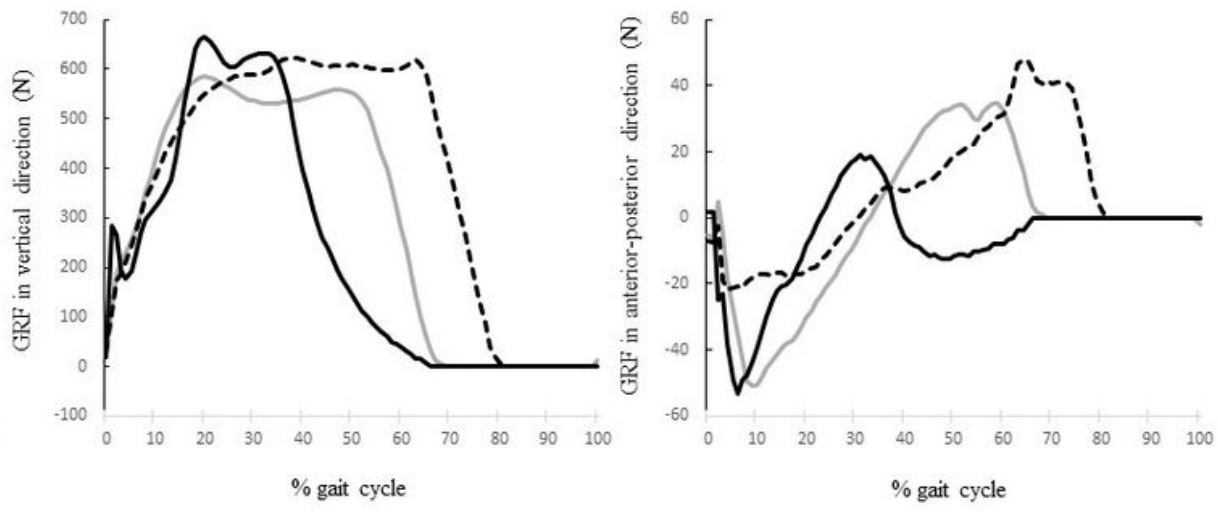

\section{Figure 1}

A typical first PC1 and GRFs in the vertical and anteroposterior directions during gait. The left, middle, and right figures indicate the PC1, the GRF in the anteroposterior direction, and the GRF in the vertical direction. 
Fig.2

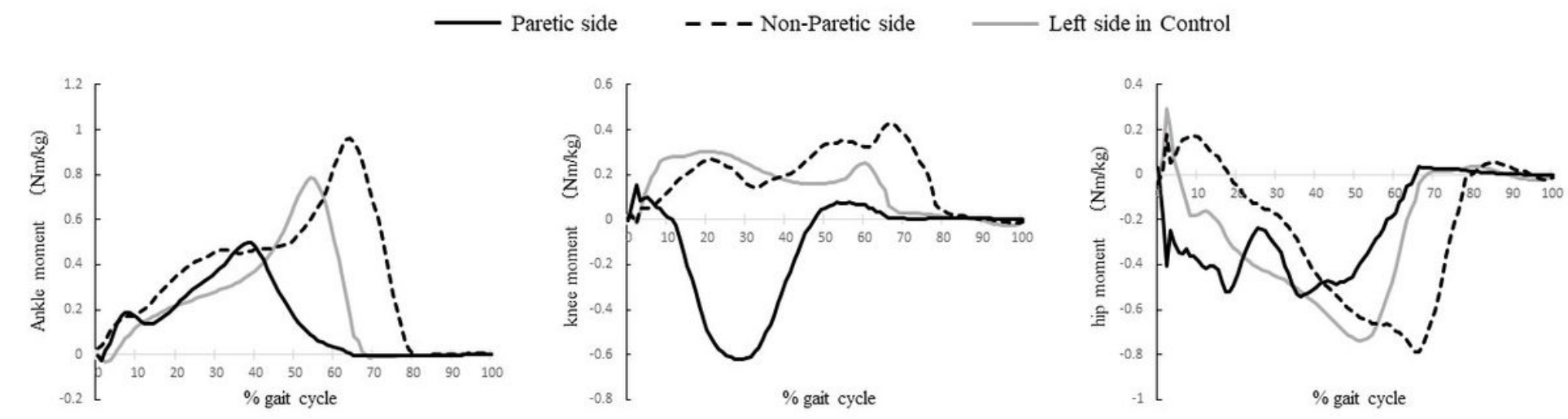

\section{Figure 2}

A typical ankle, knee, and hip moment during 1 gait cycle. The left, middle, and right figures indicate the ankle, knee, and hip moments during 1 gait cycle. 